where $S_{r}(a), S_{r}(b), S_{r}^{\prime}(a)$ and $S_{r}^{\prime}(b)$ stand for the sum of all possible combinations of $r$ factors taken at a time from the sequence of numbers $q, q^{a_{1}}, q^{a_{2}}, \ldots, g^{a_{r}}$; $q^{b_{1}}, q^{b_{2}}, \cdots, q^{b_{r}} ; q, q^{1-b_{1}}, q^{1-b_{2}}, \cdots, q^{1-b_{r}}$ and $q^{1-a_{1}}, q^{1-a_{2}}, \cdots, q^{1-a_{r}}$ respectively, we can sum up both the series on the right-hand side of (4.6) and obtain

$$
\begin{aligned}
{ }_{r} \Psi_{r}\left[\begin{array}{l}
q^{\left(a_{r}\right)} ; z \\
q^{1+\left(b_{r}\right)}
\end{array}\right]_{N}^{M}=\frac{\left[1-q^{N+1}\right]\left[q^{\left(a_{r}\right)} ; N+1\right]}{[z-1]\left[q^{1+\left(b_{r}\right)} ; N\right]} \\
+\frac{\left[1-q^{M+1}\right]\left[q^{-\left(b_{r}\right)} ; M+2\right]}{\left[q^{r+\Sigma\left(b_{r}\right)-\Sigma\left(a_{r}\right)}-z\right]} \times \frac{q^{(M+2)\left[r+\Sigma\left(b_{r}\right)-\Sigma\left(a_{r}\right)\right]}}{\left[q^{1-\left(a_{r}\right)} ; M+1\right] z^{M+1}}
\end{aligned}
$$

under the set of conditions (4.7).

On the other hand, if in the set of conditions (7a) and (7b) we assume all the conditions except those corresponding to $k=1$ and $t=1$ we can still find the sum of $M+N+1$ terms of a bilateral hypergeometric function by using the result (5).

Department of Mathematics

Lucknow University

Lucknow, India

1. L. Carlitz, "The sum of the first $n$ terms of an ${ }_{5} F_{4}$," Boll. Un. Mat. Ital., (3), v. 19, 1965 , pp. 436-440. MR 30 * 4993 .

2. T. M. MACROBERT \& F. M. RAGAB, " $E$-function series whose sums are constants." Math. Z., v. 78, 1962, pp. 231-233. MR $27 * 354$. 1966.

3. L. J. Slater, Generalised Hypergeometric Functions, Cambridge Univ. Press, London,

4. A. Verma, "A class of expansions of $G$-functions and the Laplace transform," Math. Comp., v. 15, 1965, pp. 661-664.

\title{
The Fast Fourier Transform Recursive Equations for Arbitrary Length Records
}

\section{By G. D. Bergland}

1. Introduction. In Cooley and Tukey's paper on the Fast Fourier Transform [1], a procedure was suggested which allows operation on a time series of length $N$, where $N$ is the product of an arbitrary number of integers greater than 1 . An alternate procedure is reported in this paper which has the advantage of leading directly to a set of recursive equations for this general case. The notation and methods used have been patterned as much as possible to those of Cooley and Tukey so that the reader can go directly from their paper to this one. These results were noted and used by the author in his Ph.D. dissertation [2].

2. The Recursive Equations when $N=r_{1} r_{2} \cdots r_{m}$. Consider the problem of evaluating a complex Fourier series of the form

$$
X(j)=\sum_{k=0}^{N-1} A(k) W^{j k}
$$

where $W=e^{2 \pi i / N}, j=0,1,2, \cdots, N-1$.

First $j$ and $k$ must be expressed in the following variable radix representation.

Received August 24, 1966. 


$$
\begin{aligned}
& j=j_{m-1}\left(r_{1} r_{2} \cdots r_{m-1}\right)+j_{m-2}\left(r_{1} r_{2} \cdots r_{m-2}\right)+\cdots+j_{1} r_{1}+j_{0}, \\
& k=k_{m-1}\left(r_{2} r_{3} \cdots r_{m}\right)+k_{m-2}\left(r_{3} r_{4} \cdots r_{m}\right)+\cdots+k_{1} r_{m}+k_{0} .
\end{aligned}
$$

The ranges on the variables are chosen to give a unique representation of each decimal integer (e.g. $j_{0}=0,1, \cdots, r_{1}-1 ; j_{1}=0,1, \cdots, r_{2}-1 ; k_{0}=0,1, \cdots$, $r_{m}-1 ; k_{1}=0,1, \cdots, r_{m-1}-1$; etc.). This allows (1) to be written as

$$
X\left(j_{m-1}, j_{m-2}, \cdots, j_{1}, j_{0}\right)=\sum_{k_{0}} \sum_{k_{1}} \cdots \sum_{k_{m-1}} A\left(k_{m-1}, k_{m-2}, \cdots, k_{0}\right) W^{j k} .
$$

Note that

$$
W^{j k}=W^{j\left(k_{m-1}\left(r_{2} r_{3} \cdots r_{m}\right)+\cdots+k_{0}\right]}
$$

but

$$
W^{j k_{m-1}\left(r_{2} r_{3} \cdots r_{m}\right)}=W^{\left[j_{m-1}\left(r_{1} r_{2} \cdots r_{m-1}\right)+\cdots+j_{0}\right]\left[k_{m-1}\left(r_{2} r_{3} \cdots r_{m}\right)\right]} .
$$

When the product in the exponent is formed, the term may be expressed in the following form

$$
W^{j k_{m-1}\left(r_{2} r_{3} \cdots r_{m}\right)}=\left[W^{\left(r_{1} r_{2} \cdots r_{m}\right)}\right]^{\left[j_{m-1}\left(r_{2} r_{3} \cdots r_{m-1}\right)+\cdots+j_{1}\right] k_{m-1}} W^{j_{0} k_{m-1}\left(r_{2} \cdots r_{m}\right)} .
$$

Note that $r_{1} r_{2} r_{3} \cdots r_{m}=N$ and

$$
W^{N}=\left(e^{2 \pi i / N}\right)^{N}=1,
$$

therefore the bracketed term of (6) taken to any power is still equal to 1 and we have

$$
W^{j k_{m-1}\left(r_{2} r_{3} \cdots r_{m}\right)}=W^{j 0 k_{m-1}\left(r_{2} r_{3} \cdots r_{m}\right)}
$$

therefore

$$
W^{j k}=W^{j_{0} k_{m-1}\left(r_{2} \cdots r_{m}\right)} W^{j\left[k_{m-2}\left(r_{3} \cdots r_{m}\right)+\cdots+k_{0}\right]} .
$$

This allows (3) to be written in the form

$$
\begin{aligned}
X\left(j_{m-1}, \cdots, j_{0}\right)= & \sum_{k_{0}} \sum_{k_{1}} \cdots \sum_{k_{m-2}}\left[\sum_{k_{m-1}} A\left(k_{m-1}, \cdots, k_{0}\right) W^{j_{0} k_{m-1}\left(r_{2} \cdots r_{m}\right)}\right] \\
& \cdot W^{j\left[k_{m-2}\left(r_{3} \cdots r_{m}\right)+\cdots+k_{0}\right]} .
\end{aligned}
$$

If the expression in brackets is written as

$$
A_{1}\left(j_{0}, k_{m-2}, \cdots, k_{0}\right)=\sum_{k_{m-1}} A\left(k_{m-1}, \cdots, k_{0}\right) W^{j_{0} k_{m-1}\left(r_{2} \ldots r_{m}\right)},
$$

(10) may be expressed as

$$
\begin{aligned}
X\left(j_{m-1}, \cdots, j_{0}\right)= & \sum_{k_{0}} \sum_{k_{1}} \cdots \sum_{k_{m-2}} A_{1}\left(j_{0}, k_{m-2}, \cdots, k_{0}\right) \\
& \cdot W^{j\left(k_{m-2}\left(r_{3} \cdots r_{m}\right)+\cdots+k_{0}\right)} .
\end{aligned}
$$

By applying (7) again we see that

$$
W^{j k_{m-2}\left(r_{3} r_{4} \cdots r_{m}\right)}=W^{\left(\rho_{1} r_{1}+j_{0}\right) k_{m-2}\left(r_{3} r_{4} \cdots r_{m}\right)} .
$$

This allows the innermost sum to be written as

$$
\begin{aligned}
& A_{2}\left(j_{0}, j_{1}, k_{m-3}, \cdots, k_{0}\right) \\
& \quad=\sum_{k_{m-2}} A_{1}\left(j_{0}, k_{m-2}, \cdots, k_{0}\right) W^{\left(j_{1} r_{1}+j_{0}\right) k_{m-2} r_{8} r_{4} \cdots r_{m}},
\end{aligned}
$$


leaving ${ }^{*}(12)$ in the form

$$
\begin{aligned}
X\left(j_{m-1},\right. & \left.\cdots, j_{0}\right) \\
& =\sum_{k_{0}} \sum_{k_{1}} \cdots \sum_{k_{m-3}} A_{2}\left(j_{0}, j_{1}, k_{m-3}, \cdots, k_{0}\right) W^{j\left[k_{m-3}\left(r_{4} r_{5} \cdots r_{m}\right)+\cdots+k_{0}\right]} .
\end{aligned}
$$

Proceeding in a similar fashion, a set of recursive equations are obtained of the form

$$
\begin{aligned}
A_{p}\left(j_{0}, j_{1}, \cdots, j_{p-1}, k_{m-p-1}, \cdots, k_{0}\right) & \\
= & \sum_{k_{m-p}} A_{p-1}\left(j_{0}, j_{1}, \cdots, j_{p-2}, k_{m-p}, \cdots, k_{0}\right) \\
& \cdot W^{\left[j_{p-1}\left(r_{1} r_{2} \cdots r_{p-1}\right)+\cdots+j_{0}\right] k_{m-p}\left(r_{p+1} \cdots r_{m}\right)}, \quad p=1,2, \cdots, m .
\end{aligned}
$$

Note that the last array calculated gives the Fourier sums as

$$
X\left(j_{m-1}, \cdots, j_{0}\right)=A_{m}\left(j_{0}, \cdots, j_{m-1}\right) .
$$

It should be noted that an alternate set of recursive equations is obtained if the $j k$ product is combined by expanding $j$ instead of $k$. If we represent

$$
W^{j k}=W^{j_{0} k} W^{\left[j_{1} r_{1}+j_{2}\left(r_{1} r_{2}\right)+\cdots+j_{m-1}\left(r_{1} \cdots r_{m-1}\right)\right] k}
$$

a set of $\hat{A}_{1}$ summations can be defined as

$$
\hat{A}_{1}\left(j_{0}, k_{m-2}, \cdots, k_{0}\right)=\sum_{k_{m-1}} A\left(k_{m-1}, \cdots, k_{0}\right) W^{j_{0}\left[k_{m-1}\left(r_{2} r_{3} \ldots r_{m}\right)+\cdots+k_{0}\right]} .
$$

After making use of $(7)$ the $\hat{A}_{2}$ summations can be defined as

$$
\hat{A}_{2}\left(j_{0}, j_{1}, k_{m-3}, \cdots, k_{0}\right)=\sum_{k_{m-2}} \hat{A}_{1}\left(j_{0}, k_{m-2}, \cdots, k_{0}\right) W^{j_{1} r_{1}\left[k_{m-2}\left(r_{3} \cdots r_{m}\right)+\cdots+k_{0}\right]} \text {. }
$$

The general form of these recursive equations is

$$
\begin{aligned}
& \hat{A}_{p}\left(j_{0}, j_{1}, \cdots, j_{p-1}, k_{m-p-1}, \cdots, k_{0}\right) \\
& =\sum_{k_{m-p}} \hat{A}_{p-1}\left(j_{0}, j_{1}, \cdots, j_{p-2}, k_{m-p}, \cdots, k_{0}\right) \\
& \quad \cdot W^{j_{p-1}\left[k_{m-p}\left(r_{p+1} \cdots r_{m}\right)+\cdots+k_{0}\right]\left(r_{1} \cdots r_{p-1}\right)}, \quad p=1,2, \cdots, m,
\end{aligned}
$$

where again the last array calculated gives the Fourier sums as

$$
X\left(j_{m-1}, \cdots, j_{0}\right)=\hat{A}_{m}\left(j_{0}, \cdots, j_{m-1}\right) .
$$

3. Discussion of Results. Note that (16) can be easily specialized to the recursive equations for $N=2^{m}$ which Cooley and Tukey reported. Also, their upper bound of $N\left(r_{1}+r_{2}+\cdots+r_{m}\right)$ operations to compute $N$ spectral estimates can be applied to either (16) or (21). Of course this bound can be reduced in cases where the symmetries of the complex exponential weights can be exploited.

Bell Telephone Laboratories, Incorporated Whippany, New Jersey

1. J. W. Cooley \& J. W. Tukey, "An algorithm for the machine calculation of complex fourier series," Math. Comp., v. 19, 1965, pp. 297-301. MR 31 *2843.

2. G. D. BergLand, Digital Real-Time Spectral Analysis, Ph.D. Dissertation, Iowa State University, Ames, Iowa, 1966. 